\title{
O manejo odontológico do paciente bipolar em litioterapia, com ênfase para a cárie dentária, no método clínico de intervenção - estudo de casos*
}

\author{
Dental management of bipolar patients under lithium therapy, submitted to \\ the clinical method of intervention, giving special emphasis to dental caries, - \\ case study
}

\author{
Carina Schifino Robles** \\ Onofre Francisco de Quadros*** \\ Solange Bercht****
}

\begin{abstract}
RESUMO
O presente trabalho é um estudo de casos com 8 pacientes bipolares, em litioterapia do grupo do lítio, do Hospital de Clínicas de Porto Alegre. Descreve o manejo odontológico do paciente bipolar em litioterapia, com ênfase para a cárie dentária, no método clínico de intervenção. 0 trabalho dividiu-se em três fases, com intervalos de 90 dias. Na primeira fase da pesquisa realizou-se exames odontológicos e entrevista semi-estruturada para descrever o paciente quanto a sua saúde bucal e quanto a sua convivência com a doença bipolar, com a medicação e efeitos adversos. Os pacientes receberam tratamento odontológico no método clínico e sessões de educação em saúde objetivando o aumento de sua autonomia em relação à saúde bucal até o momento da alta odontológica. Na segunda fase da pesquisa foram repetidos os exames da primeira fase, analisada a adesão do paciente bipolar ao tratamento odontológico e o paciente foi novamente entrevistado quanto a sua relação com a doença e o tratamento desta. Na terceira fase da pesquisa realizou-se os exames odontológicos e as mesmas questões da segunda fase. Observou-se que os resultados dos exames odontológicos relacionaram-se com o grau de estabilização da doença bipolar. Os pacientes com os quais se obteve os melhores resultados no tratamento odontológico foram os que estavam com a doença bipolar estabilizada. Concluiu-se que dada a freqüência da doença bipolar é lícito esperar que em algum momento o cirurgião-dentista do setor público atenda pacientes bipolares; os pacientes bipolares constituem grupo populacional específico necessitando de programa odontológico específico; os programas odontológicos específicos para os bipolares devem considerar no planejamento e no agendamento as interrupções na terapia odontológica vinculadas às características da doença bipolar, é necessário repactuar as responsabilidades e a motivação para o autocuidado bucal, o que implica em consultas odontológicas mais frequientes.
\end{abstract}

\section{UNITERMOS}

Lítio, Cárie dentária, Transtorno bipolar, Odontologia em saúde pública.

\section{INTRODUÇÃO}

De acordo com o DSM - III da American Psychiatric Association, há vários tipos de distúrbios afetivos. (FELPEL, 1989) Os distúrbios afetivos são depressão, depressão sazonal, distimia, distúrbio afetivo bipolar e depressão puerperal. (MENTAL HELP, s.d.)

Os transtornos bipolares constituem uma entidade clínica que produz prejuízos significativos em vários aspectos da vida do paciente: problemas de relacionamento social, pessoal, no desempenho ocupacional (desemprego, afastamento do trabalho, perdas financeiras) e na expectativa de vida (maior risco de suicídio). (PSYCHIATRY ON LINE BRAZII, s.d.)

Estima-se que, entre 800.000 e 1.600.000 brasileiros, sofram de doença bipolar. (CALIL, s.d.) 0 risco de ter doença maníaco-depressiva é 1-2\%, representando 1-2 pessoas de cada 100 na população. As mulheres tem mania e depressão mais freqüentes que homens. A doença frequientemente inicia entre 30 e 50 anos de idade, mas pode iniciar ainda aos 15-20 anos ou aos 60-70 anos. (SCHOU, 1980)

Portanto, na prática clínica diária, o dentista pode esperar tratar pessoas que fazem uso de grande variedade de psicotrópicos, (FELPEL, 1989) bem como estabilizadores do humor como o lítio.

Pacientes que tomam lítio queixam-se de problemas de saúde bucal, especificamente cárie dentária, segundo eles, agravados com o início da litioterapia.

O HCPA mantém um Programa de Atendimento das Doenças Afetivas do Hospital de Clínicas de Porto Alegre, na forma de grupos para os doentes bipolares.

É o chamado "grupo do lítio", embora este seja conhecido como grupo do lítio, nem todos pacientes tomam lítio para estabilizar o humor, fazendo uso de outros fármacos, como anticonvulsionantes (carbamazepina ou ácido valpróico). Isto ocorre quando o paciente não apresenta condições clínicas para ser medicado com lítio como, por exemplo, insuficiência renal, ou apresenta sintomas colaterais muito intensos que impeçam a continuação da litioterapia.

A literatura aponta relações entre lítio e cárie dentária, porém com muitas discordâncias, no sentido de que se produza ou não a doença. (CORDIOLI, 1997; CURZON, 1982; CURZON, RICHARDSON E FEATHERSTONE, 1986; FELPEL, 1989; GENTIL FILHO, 1984; JEFFERSON e colaboradores, 1987; MALTHUS, LUDWIG E HEALY, 1964; MENTAL HELP, s. d.; O'CONNELL E BOWEN, 1994; OLSON, McDONALD E STOOKEY, 1979; SHAW E GRIFFITHS, 1961; TREASURE, 1981; WEGMAN e colaboradores, 1984; WISOTZKY E HEIN, 1958) Assim como a relação do lítio com saúde bucal não está bem esclarecida, também sua ação na doença bipolar carece de maiores esclarecimentos e investigações.

* Parte de dissertação apresentada como parte dos requisitos obrigatórios para obtenção do título de mestre em Saúde Bucal Coletiva.

** Mestre em Saúde Bucal Coletiva da Faculdade de Odontologia da Universidade Federal do Rio Grande do Sul.

*** Professor livre-docente de Patologia Geral e Bucodental do Departamento de Odontologia Conservadora da Faculdade de Odontologia da Universidade Federal do Rio Grande do Sul.

**** Professora Doutora do Curso de Mestrado em Saúde Bucal Coletiva da Faculdade de Odontologia da Universidade Federal do Rio Grande do Sul. 


\section{REVISÃO DE LITERATURA}

Os indivíduos que sofrem de períodos alternantes de depressão e mania, estão acometidos pela denominada doença afetiva bipolar, antigamente chamada de psicose maníaco-depressiva. (BRUNSTEIN E LEITE, 1987; FELPEL, 1989; SCHESTATSKY E KOHLRAUSCH, s.d.).

O uso do lítio para tratamento de sintomas maníacos agudos foi introduzido em 1949 por John Cade, psiquiatra australiano, e reinvestigado por Mogens Schou, em 1952, que vem difundindo, desde então, seu uso para controle dos distúrbios afetivos, sobretudo da doença bipolar. (WANNMACHER, 1992)

A taxa terapêutica pode variar em média, entre 0,6 a $0,8 \mathrm{mEq} / \mathrm{l}$, como mínima e de 1,0 a 1,2 mEq/l, como máxima. Em idosos, os efeitos terapêuticos já podem ser obtidos com taxas de 0,4 a $0,5 \mathrm{mEq} /$. Às vezes a taxa pode ir até $1,5 \mathrm{mEq} /$, como no caso do tratamento do período agudo de mania. (BRUNSTEIN E LEITE, 1987)

0 tratamento medicamentoso não é muito simples, exige combinações de medicamentos diferentes e reajustes freqüentes de doses. (MENTAL HELP, s.d.)

O lítio não cura a doença afetiva, mas, em geral, a mantém sob controle e evita o surgimento de novas crises como em muitas doenças crônicas, nas quais o paciente sempre deve tomar a medicação. (BRUNSTEIN E LEITE, 1987)

$O$ conhecimento acerca dos efeitos adversos ao lítio é da mais alta importância, entre outras coisas, por ser esta uma terapêutica de duração indeterminada. Estes efeitos podem ser de aparecimento imediato ou tardio. (LESSA, 1984)

Os efeitos colaterais e a toxicidade tornam-se crescentemente evidentes a doses que resultam em níveis sorológicos maiores. Uma recente revisão da literatura revela que 35 a $93 \%$ dos pacientes reclamam de efeitos colaterais adversos da litioterapia. Os efeitos adversos mais comuns são sede excessiva, poliúria, problemas de memória, tremores, ganho de peso, cansaço/sonolência, diarréia, entre outros. (LENOX E MANJI, 1998)

Entre os efeitos adversos do hítio está descrito sede, polidpsia, aumento de peso, secura da boca. (KOROLKOVAS, 1997)

Descrito como reações adversas mais comuns: acne, aumento do apetite, edema, fezes amolecidas, ganho de peso, gosto metálico, náuseas, polidpsia, poliúria, tremores finos. Como reações adversas menos comuns: cáries dentárias, diabetes insipidus, hipotireoidismo. (CORDIOLI, 1997)

- A maioria dos pacientes em uso do Ltio sofre de uma disfunção das glândulas salivares e consequentemente diminuição do fluxo salivar. A polidpsia é comum, provavelmente, devido a xerostomia induzida pelo lítio." (FELPEL, 1989)

Boca seca é uma queixa comum sendo é geralmente secundária a poliúria induzida por lítio, embora haja algumas evidências de que o lítio possa também exercer efeitos determinantes na sede, podendo diminuir o fluxo salivar. (JEFFERSON e colaboradores, 1987)

“Deve-se ter cuidados higiênicos com a boca e dentes, pois o lítio, sendo também eliminado pela saliva, pode aumentar o risco de cáries." (BRUNSTEIN E LEITE, 1987) Aumentar os cuidados com a higiene bucal e dentária, pois o lítio eleva o risco do aparecimento de cáries. (CORDIOLI, 1997)

Não existe comprovação se o lítio realmente prejudica os dentes, mas se faz a mesma recomendação de boa higiene bucal que se faz com todos os pacientes medicados com antidepressivos. (MENTAL HELP, s.d.)

A formação de cáries pode ser secundária à presença de boca seca. Assim todos os fármacos que provocam algum bloqueio colinérgico podem provocar este efeito colateral e, conseqüentemente, favorecer a formação de cáries. No caso do lítio, provavelmente seja devido à sua secreção na saliva. (CORDIOLI, 1997)

Não está completamente esclarecido como e nem se o lítio afeta a taxa de fluxo salivar. (JEFFERSON e colaboradores, 1987)

\section{MATERIAIS E MÉTODOS}

Este é um estudo de casos, usado para avaliação inicial de problemas ainda mal conhecidos e cujas características ou variações não foram convenientemente detalhadas. Trata-se de observar um ou poucos indivíduos com a mesma doença ou evento e, a partir da descrição dos respectivos casos, traçar um perfil de suas principais características. (PEREIRA, 1995)

A amostra foi aleatória e constituída por 8 pacientes que aceitaram participar da pesquisa e frequientavam o grupo de lítio do HCPA, independentemente do período em que o paciente estivesse sob litioterapia.

Todos os pacientes foram informados quanto ao objetivo do estudo. Todos os pacientes assinaram um Termo de Consentimento Informado antes de participar do estudo, tendo o protocolo sido aprovado pela Comissão Científica e de Ética da Faculdade de Odontologia da UFRGS e pela Comissão Científica e Comissão de Pesquisa e Ética em Saúde do Grupo de Pesquisa e Pós-Graduação do HCPA.

A pesquisa desenvolveu-se em três fases.

Na primeira fase, foi feito o diagnóstico odontológico do paciente através dos seguintes exames: IPV, ISG, CPOS modificado, fluxo salivar, $\mathrm{pH}$ salivar, contagem de estreptococos do grupo mutans. contagem de lactobacilos, exames radiográficos, diário alimentar e entrevista objetivando descrever o paciente quanto ao nível de informação relacionado à cárie dentária, à motivação, à valorização, à conduta do paciente quanto à saúde bucal e ao tratamento clínico propriamente, que incluiu sessões de educação em saúde. objetivando o aumento da autonomia dos pacientes em relação ao controle da cárie dentária. Também analisou-se a relação do paciente com a doença bipolar e com o tratamento desta. Todas consultas odontológicas foram de 60 minutos, sendo que os primeiros 20 minutos destas consultas, quando necessários, eram usados para as sessões de educação em saúde.

Uma vez detectada a necessidade das sessões de educação em saúde, adotouse os seguintes procedimentos: conversa dirigida com o paciente sobre a evolução de seu quadro de saúde bucal e exame da boca. com espelho de toucador, explicando onde se encontravam os problemas (geralmente de placa e de sangramento), sempre se enfatizando que estávamos fazendo exame da boca de duas pessoas, ou seja, com dois olhares, tendo claro que eram duas compreensões e valorações distintas sobre o mesmo fenômeno: a saúde bucal do paciente. A conduta de examinar a dois traz implícita ou quer explicitar igualdade, se não de conhecimentos, mas de responsabilidades sobre a saúde bucal do paciente, isto é, a cada sessão, repactuou-se as responsabilidades do dentista e do paciente. no sentido de se obter melhoras, ou pelo menos, estabilização do quadro de saúde bucal.

Nas sessões de educação em saúde. discutiu-se com o paciente a etiologia da doença e seu desenvolvimento, a importância da remoção regular de placa para manutenção da saúde dentária e periodontal. o consumo de uma dieta balanceada, uso de dentifrício fluoretado e a necessidade de controle profissional regular, possibilitando. assim, ao paciente a aquisição de um conhecimento através do qual ele possa ter um certo controle sobre sua própria saúde bucal, através do autocuidado bucal.

A segunda fase foi realizada 90 dias (BRATTHALL E ERICSSON, 1995) após o final da primeira fase, e consistiu na rechamada do paciente para analisar a adesão ao tratamento odontológico, a sua relação com 
a doença bipolar e com o tratamento desta.

Os pacientes foram classificados, nesta fase, quanto à adesão ao tratamento odontológico. Considerou-se como adesão muito boa ao tratamento odontológico o paciente que diminuiu o ISG e quando questionado relatou melhora no autocuidado da boca, desde a fase anterior. Teve adesão boa ao tratamento odontológico, o paciente que diminuiu ISG, e relatou que o autocuidado ficou igual ou piorou. Com adesão ruim ao tratamento odontológico, o paciente que aumentou o ISG, e disse que seu autocuidado bucal melhorou ou ficou igual. E com adesão muito ruim ao tratamento odontológico, o paciente que aumentou o ISG, e relatou piora no autocuidado bucal.

Foram realizados os seguintes exames: CPOS modificado, IPV, ISG, fluxo salivar, pH salivar, capacidade tampão salivar, contagem de estreptococos do grupo mutans, contagem de lactobacilos e diário alimentar. Foi feita entrevista para descrever o paciente quanto ao nível de informação relacionado à cárie dentária, à motivação, à valorização e à conduta do paciente quanto à saúde bucal.

A terceira fase consistiu na segunda rechamada do paciente, ocorrida 180 dias (BRATTHALL E ERICSSON, 1995) após o final da primeira fase da pesquisa, para analisar, novamente, a adesão ao tratamento odontológico, a relação do paciente com a doença bipolar e com o tratamento desta, além de repetidos os exames CPOS modificado, IPV, ISG, fluxo salivar, pH salivar, capacidade tampão, contagem de estreptococos do grupo mutans, contagem de lactobacilos, exames radiográficos e diário alimentar. Foi feita entrevista para descrever o paciente quanto ao nível de informação relacionado à cárie dentária, à motivação, à valorização e à conduta do paciente quanto à saúde bucal. Os pacientes foram classificados, nesta fase, quanto à adesão ao tratamento odontológico

Durante a pesquisa, quando o psiquiatra solicitava a litemia sérica do paciente, foi também coletada saliva do paciente para verificar a concentração de lítio presente na saliva do paciente.

\section{RESULTADOS}

\section{LITEMIA SÉRICA E CONCENTRAÇÃO DE LÍTIO NA SALIVA}

Quando foi solicitado, pelo psiquiatra, exame de sangue para verificar a litemia, os pacientes apresentaram as litemias séricas e as quantidades de lítio na saliva que estão expressas na tabela 1 .

TABELA 1 - Distribuição da litemia sérica (expressa em $\mathrm{mEq} / \mathrm{l}$ ), da concentração de lítio na saliva (expressa em $\mathrm{mEq} / \mathrm{l}$ ) e da razão entre a concentração de lítio na saliva e a litemia sérica para os oito pacientes do grupo do lítio do HCPA, Porto Alegre, RS. 1999,

\begin{tabular}{|c|c|c|c|}
\hline Paciente & $\begin{array}{c}\text { Litemia } \\
\text { Sérica }\end{array}$ & $\begin{array}{c}\text { Concentração } \\
\text { de lítio na } \\
\text { saliva }\end{array}$ & $\begin{array}{c}\text { Razão entre a } \\
\text { concentração } \\
\text { de lítio na } \\
\text { saliva e litemia } \\
\text { sérica }\end{array}$ \\
\hline A & 1,00 & 2,00 & 2,0 \\
B & 0,90 & 2,40 & 2,6 \\
C & $*-$ & - & - \\
D & 0,90 & 1,75 & 1,9 \\
E & 090 & 2,40 & 2,6 \\
F & 1,50 & 1,80 & 1,2 \\
G & 1,40 & 1,70 & 1,2 \\
H & 1,00 & 1,70 & 1,7 \\
\hline
\end{tabular}

* 0 psiquiatra não solicitou exame de sangue para verificar a litemia enquanto a paciente $\mathrm{C}$ participava da pesquisa.

\section{RESULTADOS DOS EXAMES ODONTOLÓGICOS DO CONJUNTO DOS PACIENTES BIPOLARES QUE PARTICIPARAM DA PESQUISA}

Analisando o IPV, de um modo geral, vemos que os pacientes $\mathrm{B}, \mathrm{D}, \mathrm{E}, \mathrm{F}, \mathrm{G}$ e $\mathrm{H}$ reduziram o IPV na terceira fase quando comparado com o IPV da primeira fase; a paciente A obteve, na terceira fase IPV maior que o IPV da primeira fase e a paciente $C$ iniciou e terminou com o mesmo IPV: 0\%.

Observamos no que nos exames de ISG os pacientes A, D, E, F, G e H conseguiram reduzir o ISG da primeira fase para a terceira fase; a paciente $\mathrm{C}$ apresentou ISG igual a $0 \%$ nas três fases e apenas a paciente B mostrou ISG maior na terceira fase quando comparado com o ISG da primeira fase, sendo que este havia decrescido na segunda fase.

Verificando o CPOS modificado, notase que os pacientes $\mathrm{D}, \mathrm{E}, \mathrm{F}$ e $\mathrm{G}$ mantiveram o mesmo CPOS modificado nas três fases das pesquisa. Os pacientes A, B, C, H aumentaram o CPOS modificado.

Os índices de fluxo salivar mostram que o menor fluxo salivar entre os pacientes nas três fases da pesquisa foi $0,8 \mathrm{ml} / \mathrm{min}$, velocidade considerada como um fluxo intermediário entre o fluxo baixo e o normal, para fins deste estudo. Nenhum paciente apresentou xerostomia, isto é, segundo KRASSE (1988), fluxo menor que $0,1 \mathrm{ml} /$ min. $O$ paciente $E$ sempre obteve fluxo maior que $2,0 \mathrm{ml} / \mathrm{min}$, considerado nesta pesquisa como fluxo alto e, ainda os pacientes $\mathrm{A}$ e $\mathrm{C}$, na primeira fase, e o paciente $G$ na segunda e terceira fases também obtiveram fluxo alto.

Analisando os exames de $\mathrm{pH}$ salivar, concluímos que o $\mathrm{pH}$ dos oito pacientes variou de normal $(\mathrm{pH}=7)$ a básico ( $\mathrm{pH}$ maior que 7$)$.

Os exames de capacidade tampão demonstram que a capacidade tampão dos pacientes $\mathrm{A}, \mathrm{B}, \mathrm{C}, \mathrm{D}, \mathrm{F}$ e $\mathrm{H}$, na segunda e terceira fases manteve-se com $\mathrm{pH}$ final entre 5 e 7 , ou seja, capacidade tampão normal. Os pacientes E e $\mathrm{G}$ apresentaram na segunda fase $\mathrm{pH} 8$ e na terceira fase $\mathrm{pH}$ igual a 6 .

Depreende-se que todos pacientes foram classificados, de acordo com os resultados da contagem de estreptococos do grupo mutans, em pacientes de muito alto risco à cárie dentária na primeira fase. $\mathrm{Na}$ segunda fase os pacientes $\mathrm{D}, \mathrm{B}, \mathrm{E}, \mathrm{G}$ e $\mathrm{H}$ passaram a ser classificados como pacientes de alto risco à cárie dentária e permanecerem com esta classificação na terceira fase da pesquisa. Os pacientes $\mathrm{F}$ e $\mathrm{C}$ passaram na segunda fase da pesquisa para a classificação de baixo risco à cárie dentária e mantiveramse assim na terceira fase. A paciente A na segunda fase apresentou baixo risco à cárie dentária, porém na terceira fase foi classificada como de alto risco à cárie dentária.

Os exames de contagem de lactobacilos mostram que os pacientes $\mathrm{C}$ e $\mathrm{G}$ foram classificados como muito alto risco à cárie dentária na primeira fase e baixo risco à cárie dentária na terceira fase. $\mathrm{O}$ paciente $\mathrm{H}$, na primeira fase, foi classificado como de muito alto risco à cárie dentária; na segunda, de baixo risco à cárie dentária e, na terceira, como de alto risco à cárie dentária. Os pacientes $\mathrm{A}, \mathrm{B}, \mathrm{F}$ e $\mathrm{D}$ terminaram a pesquisa como de muito alto risco à cárie dentária, sendo que o paciente $\mathrm{B}$ na primeira fase tinha alto risco à cárie dentária e os pacientes $\mathrm{A}, \mathrm{F}$ e $\mathrm{D}$ tinham, na primeira fase baixo risco à cárie dentária. $\mathrm{O}$ paciente $\mathrm{E}$ iniciou e terminou sendo classificado como baixo risco à cárie dentária, na segunda fase, foi alto risco à cárie dentária.

Quanto à distribuição da frequência de consumo de sacarose total diário apenas os pacientes E e F, na primeira e segunda fases; e o paciente $G$, na segunda e terceira fases apresentaram 5 ou mais consumos diários de sacarose total, o que representa, nesta pesquisa alto consumo de sacarose.

\section{DISCUSSÃO}

No decorrer da pesquisa, foram se impondo algumas questões que se mostraram relevantes, e que serão objeto de discussão: a freqüência do transtorno bipolar, a doença bipolar, o fato do paciente bipolar constituir um grupo populacional específico, e o manejo deste paciente em odontologia de saúde pública.

Concorda-se com SONIS, FAZIO E 
FANG (1996), quando referem que é óbvio, que o dentista encontrará um número significativo de pacientes recebendo drogas que afetam seu estado psíquico.

Por outro lado, há que se considerar que há um número de pacientes bipolares sem diagnóstico e sem terapia farmacológica e psiquiátrica, e que, contudo, são doentes. Ou seja, têm as manifestações da doença com o comprometimento psíquico e social do paciente.

Inclusive sob o ângulo farmacológico, que poderia se tomar como uma ótica restrita, os pacientes bipolares constituem um grupo populacional específico.

Sob o prisma farmacológico, é necessário que se considere a interação das drogas psicoativas com vasoconstritores nos anestésicos locais e depressores do sistema nervoso central. Neste sentido, SONIS, FAZIO E FANG (1996), sustentam que pode haver ou não contra-indicação relativa ou absoluta para o uso destes últimos agentes, em virtude do possível sinergismo com a medicação psicoativa.

Há que se considerar que, durante a terapia do paciente bipolar, os fármacos ministrados aos pacientes podem sofrer variações em função das manifestações da doença em cada paciente, bem como em função dos efeitos colaterais da litioterapia que são individuais.

São raros os pacientes bipolares medicados exclusivamente com lítio. Entre os pacientes estudados, apenas um paciente medicava-se somente com lítio.

Os fármacos utilizados concomitantemente ao lítio, relacionados à doença bipolar podem ser antidepressivos, (BRUNSTEIN E LEITE, 1987; CORDIOLI, 1997; LENOX E MANJI, 1998; SCHOU, 1980) antipsicóticos, (BRUNSTEIN E LEITE, 1987; SCHOU, 1980) anticonvulsionantes, (CORDIOLI, 1997) e diuréticos. (LENOX E MANJI, 1998) Nos casos estudados, também havia pacientes medicados com drogas reguladoras de lipídio, regulador de tireóide e antimicótico, em função de outros agravos à saúde que podem ser decorrentes dos paraefeitos da litioterapia.

$\mathrm{Na}$ presente pesquisa, nenhum paciente, dos oito casos relatados, apresentou xerostomia, sendo que, em um caso, houve fluxo salivar alto. Segundo convenção desta pesquisa, fundada em KRASSE (1988), considera-se alto um fluxo de mais do que $2 \mathrm{ml} / \mathrm{min}$ de saliva.

A queixa de boca seca foi referida, por seis dos oito pacientes estudados, concomitante com a sensação de sede e ingesta frequiente de líquidos.

Há relatos de polidpsia, (CORDIOLI,
1997; KOROLKOVAS, 1997; LESSA, 1984) de aumenta na ingestão de água, (BRUNSTEIN E LEITE, 1987; TAHIN, 1984) de ingestão de bebidas calóricas, (SCHOU, 1980) ou de efeitos determinantes na sede, (JEFFERSON e colaboradores, 1987) como efeitos colaterais do lítio. Vários autores relatam a poliúria como efeito colateral do lítio. (BRUNSTEIN E LEITE, 1987; CORDIOLI, 1997; JEFFERSON e colaboradores, 1987; LESSA, 1984) A sensação de boca seca é uma queixa comum dos pacientes bipolares, podendo ser secundária a poliúria e polidpsia.

Portanto, corrobora-se os resultados de SREEBNY, VALDINI E BROOK, (1988) relatando que, em pacientes que reclamam de boca seca, o fluxo salivar não é prejudicado. Foi registrado fluxo salivar alto em vários pacientes nas diversas fases deste estudo corroborando estudo realizado por JEFFERSON e colaboradores (1987) que não observou alteração no fluxo salivar, ou ainda houve fluxo salivar alto.

Em relação à cárie dentária e à ingesta de lítio, a literatura também não se mostrou conclusiva, embora esteja demonstrado que o lítio é um fármaco ativamente secretado na saliva. (PASSARELLI E MORAES, 1989) Assim, CORDIOLI (1997) refere que a formação de cáries pode estar relacionada com a secreção de lítio na saliva, em função da boca seca, como efeito colateral do fármaco. BRUNSTEIN E LEITE (1987) postulam que o lítio sendo excretado na saliva pode aumentar o risco de cáries.

A literatura aponta a cárie dentária como paraefeito do lítio. (CORDIOLI, 1997) Íons de lítio administrados na água potável não afetam a incidência de cáries. (OLSON, McDONALD E STOOKEY, 1979, CURZON, 1982; WISOTZKY E HEIN, 1958) O lítio está relacionado com a redução de cáries. (CURZON, RICHARDSON E FEATHERSTONE, 1986; MALTHUS, LUDWIG E HEALY, 1964; WEGMAN e colaboradores, 1984) Constatou-se que o lítio esteve associado com a incidência aumentada de cárie. (CURZON, RICHARDSON E FEATHERSTONE, 1986)

Em relação à cárie dentária, a discussão pode ser orientada para o fato de que pacientes bipolares em presença de sensação de boca seca, aumentam a ingesta de líquidos, que podem conter açúcares, como relatam LESSA (1984) e SCHOU (1989).

Merece discussão o fato de que o lítio está presente na saliva em alta concentração, quando comparado com sua concentração no soro sanguíneo, (LAZARUS e colaboradores, 1973; SPRING E SPIRTES, 1969; PASSARELLI E MORAES, 1989) fato também observado no presente estudo, no qual a concentração de lítio na saliva foi de 1,2 a 2,6 vezes maior do que a concentração no soro sanguíneo dos pacientes estudados.

Sabe-se que o lítio é um íon extremamente reativo, univalente e que compete nos tecidos biológicos com os íons $\mathrm{Na}^{+}, \mathrm{K}^{+}, \mathrm{Ca}^{++}, \mathrm{Mg}^{++}$. (TAHIN, 1984)

Portanto, o íon lítio livre pode interferir em vários processos biológicos fons dependentes. (TAHIN, 1984) Neste sentido questiona-se se o íon lítio livre excretado na saliva, poderia interferir com os íons $\mathrm{Ca}^{++} e$ $\mathrm{F}^{+}$, competindo com o íon $\mathrm{Ca}^{++}$na formação de $\mathrm{CaF}_{2}$. Assim, levanta-se a discussão e fica em aberta a questão da resolutividade da fluorterapia em pacientes bipolares medicados com lítio.

Em face do exposto, e se verdadeira a hipótese de que a ingesta de lítio poderia interferir na fluorterapia, para aqueles pacientes nos quais ela estivesse indicada. mereceria ser estudado, por exemplo, o uso de um creme dental específico, que fosse quelante do lítio, de forma a não deixar o íon lítio livre, interferindo na presença do flúor na saliva.

Saindo da questão da ação farmacológica do lítio no meio bucal do paciente bipolar, ressalta-se a importância de discutir algumas características que os pacientes deste estudo apresentaram, e que dificultam o processo de construção da consciência sanitária, através do autocuidado.

Uma das primeiras observações, é que o paciente bipolar, embora esteja sob terapia farmacológica e de grupo, tem dificuldades em aceitar que é doente, ocorrendo a negação da doença. $O$ grau de negação da doença é um aspecto extremamente relacionado à singularidade de cada paciente, ocorrendo com maior ou menor intensidade, podendo chegar à situação limite do abandono da terapia farmacológica e de grupo, o que pode interferir na continuidade do tratamento odontológico, fato verificado neste estudo.

Assim, a negação da doença bipolar. com o abandono da terapia farmacológica $e$ de grupo, a própria revisão do diagnóstico de doença bipolar, que pode ocorrer até em função das complexidades inerentes à patologia, podem interferir na continuidade do tratamento odontológico.

Outro aspecto que interfere na continuidade do tratamento odontológico é o fato de que, no decorrer da terapia bipolar. em função das crises pelas quais o paciente passa, ocorre a internação do paciente.

Os pacientes bipolares que apresentaram crises ou sintomas de crise mais frequientes, também são aqueles que apresentaram maior dificuldades em manter o 
autocuidado com a boca. Nos períodos de crise, registraram-se transferências nas datas das consultas odontológicas previamente agendadas, o que dificulta a continuidade do tratamento odontológico curativo e educativo e a manutenção de um planejamento no caso de serviços com agendamento prévio e que adotam regras do tipo "paciente que faltar duas vezes perde a vaga ou vai para o final da fila de espera."

O paciente bipolar deste estudo demonstrou uma dificuldade de autonomia para a vida, para a construção do seu vir a ser, neste sentido necessita a ancoragem de alguém, que pode ser um familiar, ou amigo ou cuidador. Os pacientes quando questionados em relação a sua expectativa de vida, ao seu vir a ser, não conseguem verbalizar e muitas vezes não têm o que dizer a respeito, ou seja, tem-se respostas do tipo "não estou pensando nisto agora". 0 paciente como que "desqualifica" o próprio ato de pensar o futuro, dando ao interlocutor a sensação de que há um grande vazio em relação a esta questão. Neste estudo verificouse que é comum que o bipolar não trabalhe, não tenha gerência sobre suas finanças, que, às vezes, até inexistem, sendo sustentados pela família.

Observou-se que o estado de compensação da doença bipolar relacionase diretamente com a possibilidade de autonomia do paciente sobre o próprio corpo, no caso, sobre o autocuidado com a saúde bucal. Assim, levar em conta o paciente bipolar como um todo, é de importância para o próprio manejo odontológico do paciente, o que ocorreu neste estudo, no qual a melhora nos índices IPV e ISG e adesão ao tratamento odontológico, caracterizada como boa a muito boa, espelham estes fatos.

Quanto aos exames de pH salivar, capacidade tampão salivar e microbiológicos de contagem de estreptococos do grupo mutans e lactobacilos, tem-se que os de $\mathrm{pH}$ salivar e capacidade tampão salivar ficaram nos limites de normalidade.

Contudo, registrou-se uma grande oscilação quanto à contagem de lactobacilos nas três fases da pesquisa, julgando que tal fato tenha ocorrido pela variação da ingesta de carboidratos (BRATTHALL E ERICSSON, 1995; MALTZ E CARVALHO, 1997), talvez relacionada com a instabilidade inerente a doença bipolar.

O mesmo raciocínio pode-se aplicar a contagem de estreptococos do grupo mutans que permaneceu alta em 6 dos 8 pacientes estudados, inobstante a adequação bucal, remetendo à questão da ingesta de sacarose. (MALTZ E CARVALHO, 1997)

A evolução da saúde bucal do paciente bipolar relaciona-se com a própria evolução da doença bipolar, vinculando-se aos aspectos cíclicos da patologia bipolar, não podendose afirmar definitivamente que este tipo de paciente consiga uma regularidade e uma estabilidade no quadro de saúde bucal, embora os resultados satisfatórios registrados nesse sentido.

Se para os outros pacientes que não os bipolares, a resolutividade no método clínico de intervenção é importante, para os pacientes bipolares ela é fundamental, pois constitui uma das pontes de confiança do paciente para com o profissional e o serviço, facilitando o processo de adesão à terapia odontológica.

Assim, trabalhar com a noção de integralidade e resolutividade atendendo as necessidades sentidas do paciente, não só em relação à cárie dentária, mas tratando da saúde bucal como um todo, conseguiu-se, com a maioria dos pacientes desta pesquisa, graus de adesão de muito bom a bom, que foi mantido também pela freqüência com que o paciente vinha às consultas odontológicas.

A questão do vínculo afetivo e do acolhimento mostrou-se essencial para este tipo de paciente, que, na maioria das vezes, demonstra carência afetiva, falta de entrosamento familiar em função da não aceitação da doença pelos seus familiares, pelo sofrimento psíquico ao qual a doença os submete e pela dificuldade que o bipolar apresenta nas relações com o mundo social.

Portanto, pelo que se observou neste estudo, um programa odontológico para paciente bipolar deverá contar com cirugiõesdentistas que tenham um mínimo de conhecimento sobre a doença bipolar, sobre a litioterapia e seus efeitos adversos, e sobre as características do paciente bipolar, permitindo um manejo de integralidade. Estes profissionais deveriam ser sempre os mesmos, na medida em que servem de referência afetiva para o paciente bipolar.

Questiona-se se, no setor público, no qual, a excelência do método clínico tem sido relegada a segundo plano, principalmente nos aspectos de resolutividade, de integralidade das ações e de acolhida dos pacientes, é viável o atendimento deste grupo populacional específico: o paciente bipolar. Na falta de setores de odontologia de saúde pública que saibam manejar este paciente imagina-se que os mesmos irão engrossar a fila dos pacientes que simplesmente abandonam o tratamento odontológico pela metade ou apenas o procuram em caso de dor.

\section{CONCLUSÃO}

Dada a frequiência da doença bipolar diagnosticada e considerando os casos não diagnosticados é lícito esperar que, em algum momento, o cirurgião-dentista do setor público atenda pacientes bipolares.

Os pacientes bipolares devem ser tratados com um grupo populacional específico necessitando de um programa odontológico específico, no qual o cirurgiãodentista conheça as características da doença bipolar, a terapêutica medicamentosa, a farmacologia do lítio e seus efeitos adversos.

Pelas próprias características dos pacientes, os profissionais deveriam ser sempre os mesmos para atender os pacientes deste grupo populacional específico, facilitando o vínculo de confiança e afetivo indispensável ao paciente bipolar.

$\mathrm{Na}$ elaboração de programa odontológico específico para os pacientes bipolares deve-se considerar, no planejamento e no agendamento destes pacientes, as interrupções na terapia odontológica, vinculadas às características da doença bipolar, que, às vezes, impedem a continuidade do tratamento.

O paciente bipolar necessita uma repactuação de responsabilidades e de motivação para o autocuidado bucal, o que implica consultas odontológicas mais freqüentes se comparado ao paciente normal, fato que deve ser analisado no planejamento de programas específicos.

Os pacientes com os quais se obteve melhores resultados no tratamento odontológico foram aqueles que estavam com a doença bipolar estabilizada, fato que se confirma, pois vários pacientes que participaram da pesquisa, passaram do grupo do lítio com frequiência quinzenal para a frequiência mensal. Os pacientes que tinham a doença menos compensada não terminaram as três fases da pesquisa.

A introdução do controle químico das doenças bucais, mesmo quando indicado, deve ser muito bem avaliada em função da excreção de lítio na saliva do paciente bipolar, inclusive, em concentração maior do aquelas registradas no soro sanguíneo.

\section{SUMMARY}

The present work is a case study of 8 bipolar patients under lithium therapy, being all of them members of the lithium group of the Hospital de Clínicas de Porto Alegre. It describes the dental management of bipolar patients under lithium therapy, emphasizing dental caries and the clinical method of intervention. The work was divided into three phases, existing a ninety-day interval between each of them. In the first part of the research, dental examinations were carried out and the patients were interviewed with the special purpose of describing each one of them regarding his buccal health. They were also 
inquired about their experience in living with the bipolar disease, about the medication taken and its side effects. The patients received dental treatment according to the clinical method as they also attended health educational sessions destined to increase their autonomy up to the dental dismissal. In the second and third phases, the exams that had been carried out in the first phase were repeated in order to analyze the commitment of each bipolar patient to the proposed dental treatment. The questions about the relationship between the bipolar patient and the treatment of this disease were also repeated. It was concluded that the results of the dental exams were related to the degree of stabilization of the bipolar disease; the patients detaining the best results in the dental treatment were those who had the bipolar disease stabilized. It was concluded that, having in mind the occurrence frequency of bipolar disease, it would be plausible that a dentist who works in the Public Service sooner or later would attend bipolar patients; bipolar patients are a peculiar populational group that need a specific dental program; the planning and scheduling of these specific odontological programs for bipolars should take into account the disruptions in the dental therapy, caused by the disease and it is also necessary to renegotiate with the bipolar, his responsibilities and his motivation for buccal self-care, which will result in more frequent dental visits.

\section{KEYWORDS}

Lithium, Dental caries, Bipolar disorders, Dentistry in Public Health.

\section{REFERENCIAS BIBLIOGRÁFICAS}

1. BRATTHALL, D.; ERICSSON, D.

Testes para determinar o risco de cárie. In: THYLSTRUP, A., FEJERSKOV. O. Cariologia clínica. 2. ed. São Paulo : Santos, 1995. Cap. 16, p. 333-354.

2. BRUNSTEIN, B.; LEITE, F. A. Manual para o uso do lítio. Porto Alegre, 1987.

3. CALIL, H. M. (coord.) Transtorno bipolar: informações ao paciente e familiares. São Paulo: Associação Brasileira de Psiquiatria, Novartis. s.d. 15p.

4. CORDIOLI, A. V. Psicofármacos consulta rápida. Porto Alegre: Artes Médicas, 1997. 316 p.

5. CURZON, M. E. J. An experimental study of lithium and dental caries in the rat. Arch. Oral. Biol., Oxford, v. 27, p. $573-576.1982$

6. CURZON, M. E. J., RICHARDSON, D. S.; FEATHERSTONE, J. D. B. Dental caries prevalence in Texas schoolchildren using water supplies with high and low lithium and fluoride. J. Dent. Res., Alexandria, v. 65, n. 3, p. 421-423, mar. 1986.

7. FELPEL, L. Psicofarmacologia. In: NEIDLE, E. A. Farmacologia e terapêutica para dentistas. 3. ed. Rio de Janeiro: Guanabara Koogan, 1989. Cap.12, p. 130-142

8. GENTIL FILHO, V. Alterações dentárias. J. Bras. Psiq., São Paulo, v. 33, n. 6, p. 442-446, nov./dez. 1984.

9. JEFFERSON, J. W. etal. Lithium encyclopedia for climical practice. $2 \mathrm{ed}$. Washington : American Press Psychiatry, 1987.

10.

KOROLKOVAS,

A.

Dicionário terapêutico guanabara edição 97/98. Rio de Janeiro: Guanabara Koogan, 1997.

11.

KRASSE, B. Risco de cáries: guia prático para controle e assessoramento. 2. ed. São Paulo : Quintessence, 1988.

12. LAZARUS, J. H. Secretion of lithium in human parotid saliva in maniac depressive patients treated with lithium carbonate. Arch. Oral. Biol., Oxford, v. 18, p. 329-335. 1973.

13.

LENOX, R. H., MANJI, H. K. Lithium. In: SCHATZBERG, A. F., NEMEROFF, C. B. Textbook of psychopharmacology. 2 ed. Washington: American Psychiatric Press, 1998. Cap. ,p. 379-429.

14.

LESSA, L. M. Efeitos adversos mais comuns no curso da litioterapia. J. Bras. Psiq., São Paulo, v. 33, n. 6, p. 431-433, nov./dez. 1984.

15. MALTHUS, R. S.; LUDWIG, T. G.; HEALY, W. B. Effect of trace elements on dental caries in rats. N. Z. Dent. J., Auckland, v. 60, p. 291-297, Oct. 1964.

16.

MALTZ, M.; CARVALHO,

J. Diagnóstico da doença cárie. In: KRÜGER, Léo (coord.). ABOPREV: promoção de saúde bucal. São Paulo : Artes Médicas, 1997. Cap. 4, p. 69-91.

\section{7. "MENTAL HELP."} $<$ http://www.mentalhelp.com.br $>$ [09/06/99].

18.

O'CONNELL, A.C.; BOWEN, W.H. Composition and flow rate of saliva and caries development in young rats following administration of lithium. Caries Res., Brasel, v. 28, p. 342-347, Feb. 1994.

19.

OLSON, B. L., McDONALD Jr, J. L; STOOKEY, G. K. Influence of lithyum upon dental caries in the rat. J. Dent. Res., Alexandria, v. 58, n. 4, p. 1428, Apr. 1979.

20.

PASSARELLI, M. M.; MORAES, E. C. F., Saliva como amostra para o controle terapêutico do lítio. Rev. Fárm. Bioq. Univ. São Paulo, São Paulo, v.25, n. 1, p. 71-85, jan./jun. 1989.

21.

PEREIRA, M. G.

Epidemiologia teoria e prática. Rio de Janeiro: Guanabara Koogan, 1995. Cap. 12, p. 269-288: Métodos empregados em epidemiologia.

22.

"PSYCHIATRY ON LINE

BRAZIL." < http://www.priory.com/psych/ litgui.htm $>$ [03/06/99]

23. SCHESTATSKY, S., KOHLRAUSCH, E. Manual de doenças afetivas: orientação para familiares e pacientes do programa de atendimento das doenças afetivas. Porto Alegre: Hospital de Clínicas de Porto Alegre, s.d.

24.

SCHOU, M. Lithium

treatment of manic-depressive illness, a pratical guide. Switzerland: S. Karger, 1980. $72 \mathrm{p}$.

25.

SCHOU, M. Lithium prophylaxis: myths and realities.Am. J. Psychiatry, Washington, v. 146, n. 5, p. 573-576, May. 1989.

26. SHAW, J. H., GRIFFITHS, D. Developmental and postdevelopmental influences on incidence of experimental dental caries resulting from dietary supplementation by various elements. Arch. Oral. Biol. Oxford, v. 5, p. 301322. 1961.

27.

SONIS, S.; FAZIO, R.; FANG, L. Princípios e prática de medicina oral. 2 ed. Rio de Janeiro: Guanabara Koogan, 1996. Cap.34, p.291293: Doença psiquiátrica.

28. SPRING, K. R.; SPIRTES, M. A. Salivary excretion of lithium: I. Human parotid and submaxillary secretions. J. Dent. Res., Alexandria, v. 48, n. 4, p.546549,Aug. 1969.

29.

SREEBNY, L. M.; VALDINI, A.; BROOK, S. Xerostomia. Part: relationship to other oral symptons and salivary gland hypofunction. Oral Surg. Oral Med. Oral Pathd. Oral Radiol Endod., St. Louis, v. 66, n. 4, p.451-458, Oct. 1988. 30 .

TAHIN, Q. S. Lítio e o metabolismo de carboidratos e lipídios. J. Bras. Psiquiatria, São Paulo, v. 33, n. 6, p. 418-421, nov./dez. 1984.

31. TREASURE, P. Effects of fluoride, lithium and strontium on extracellular polysaccharide production by streptococcus mutans and actinomyces viscosus. J. Dent. Res., Alexandria, v. 60(C), p. 1601-1610, Aug. 1981.

32.

WANNMACHER, L。 
Fármacos usados nos distúrbios afetivos. In: FUCHS, F. D.; WANNMACHER, L. Farmacologia clínica: fundamentos da terapêutica racional. Rio de Janeiro : Guanabara Koogan, 1992. Cap. 30, p. 347-358.

33.

WEGMAN, M.R., et al.

Effects of fluoride, lithium and strontium on intracellullar polysaccharide accumulation in $S$. mutans and A. viscosus. J. Dent. Res., Alexandria, v. 33, n. 9, p. 1126-1129, Sept. 1984.

34. WISOTZKY, J.; HEIN, J. W. Effects of drinking solutions containing metallic ions above and below hydrogen in the electromotive series on dental caries in the syrian hamster. J. Am. Dent. Assoc., Chicago, v. 57, p. 796-800, Dec. 1958.

Endereço para correspondência: Solange Bertch / Carina Schifino Robles Faculdade de Odontologia da UFRGS Centro de Pesquisas em Odontologia Social Rua Ramiro Barcelos, 2492 - $4^{\circ}$ andar, sala 402 B

Porto Allegre / RS

CEP 90035-003

e-mail: cpos@orion.ufrgs.br 